\title{
ALGORITHMIC FORMULATION AND NUMERICAL IMPLEMENTATION OF COUPLED ELECTROMAGNETIC-INELASTIC CONTINUUM MODELS FOR ELECTROMAGNETIC METAL FORMING
}

\author{
* University of Dortmund, \\ Chair of Scientific Computation, \\ 44221 Dortmund, Germany \\ e-mail: stiemer@math.uni-dortmund.de \\ e-mail: blum@math.uni-dortmund.de \\ $\dagger$ University of Dortmund, \\ Chair of Mechanics \\ 44221 Dortmund, Germany \\ e-mail: unger@mech.mb.uni-dortmund.de \\ e-mail: svendsen@mech.mb.uni-dortmund.de
}

Marcus Stiemer*, Jaan Unger ${ }^{\dagger}$, Bob Svendsen $^{\dagger}$ and Heribert Blum*

Key words: Continuum mechanics; electromagnetic-inelastic coupling; multifield problems; finite element method

\begin{abstract}
Electromagnetic metal forming is a contact-free high-speed forming process in which strain rates of more than $10^{3} \mathrm{~s}^{-1}$ are achieved. The deformation of the workpiece is driven by a material body force, the Lorentz force, that results from the interaction of a pulsed magnetic field with eddy currents induced in the workpiece by the magnetic field itself. The purpose of this work is to present a fully-coupled $3 D$ simulation of the process. For the mechanical structure a thermoelastic, viscoplastic, electromagnetic material model is relevant, which is incorporated in a large-deformation dynamic formulation. The evolution of the electromagnetic fields is governed by Maxwell's equations under quasistatic conditions. Their numerical solution in $3 D$ requires particular arrangements due to a reduced regularity at material interfaces. Hence, Nédélec elements are employed. Coupling between the thermomechanical and electromagnetic subsystems takes the form of the Lorentz force, the electromotive intensity, and the current geometry of the workpiece. A staggered scheme based on a Lagrangian mesh for the workpiece and an ALE formulation for the electromagnetic field is utilized to solve the coupled system, guaranteeing the efficiency and accuracy of the data transfer between the two meshes.
\end{abstract}

\section{INTRODUCTION}

Electromagnetic metal forming (EMF) is a contact-free high-speed forming process in which strain rates of more than $10^{3} \mathrm{~s}^{-1}$ are achieved. In this process, the deformation 
of the workpiece is driven by a material body force, the Lorentz force, that results from the interaction of a pulsed magnetic field with eddy currents induced in the workpiece by the magnetic field itself. The magnetic field is triggered by a tool coil adjacent to the workpiece, which is excited by the discharging current of a capacitor bank. Figure 1 displays a typical device for sheet metal forming. EMF offers certain advantages over
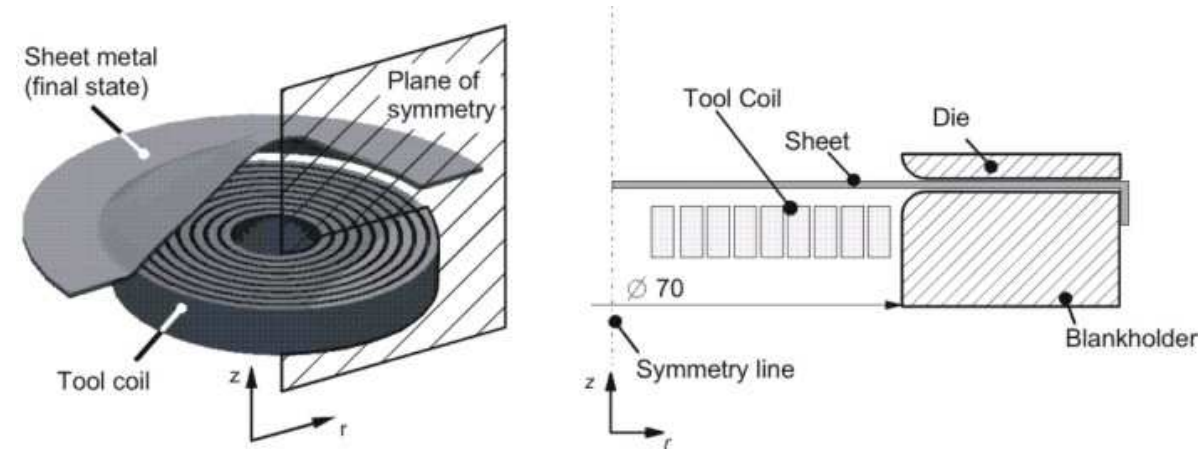

Figure 1: A typical device for electromagnetic sheet metal forming.

other forming methods such as an increased formability, the avoidance of contact, a reduction in wrinkling, reduced tool making costs, the opportunity to combine forming and assembly operations, and many more. However, the highly dynamic nature of this process inhibits its monitoring and control. Consequently, its industrial use has been limited to joining tubular semi-finished materials, while e.g., electromagnetic sheet metal forming is not ready for a profitable application yet. This emphasizes the significance of reliable simulations of this process to identify relevant process parameters and to optimize them.

Since the introduction of high speed computers in the 1980s, several attempts at the numerical simulation of EMF have been undertaken ${ }^{1,2,3,4}$. More recently, Beerwald et al. ${ }^{5}$ and Brosius et al. ${ }^{6}$ utilized commercial programs like ABAQUS or MARC for the simulation of the process. However, in all approaches reported on above emphasis is placed on the modeling and simulation of the coupling between the electromagnetic and the mechanical model, while the employed material models were not adapted to the particular requirements of the process. These include first of all a consideration of the rate-dependence, which is typical of the behavior of metallic materials at high forming rates such as those achieved during EMF. This is connected to the fact that the mechanical dissipation may result in a possibly significant temperature increase in this nearly adiabatic process. Recently, a relevant thermodynamically-consistent electromagnetic thermoelastic multifield model has been developed ${ }^{7,8}$ and implemented ${ }^{9}$ based on a Lagrangian formulation for the mechanical system and an Eulerian formulation on a fixed mesh for the electromagnetic system within an axisymmetric context.

A drawback of the numerical schemes reported on above is their restriction to twodimensional or axisymmetric situations. However, practical forming devices often significantly deviate from axisymmetry. Beside the much larger number of unknowns that 
dramatically increase the numerical expenses necessary to solve the problem and that require much more sophistication to avoid unacceptably long computing times, threedimensional electromagnetic simulations demand a particular numerical treatment due to the lack of smoothness solutions to Maxwell's equations typically exhibit at material interfaces (see section 3). There are several methods to cope with this problem, including penalty or least square approaches ${ }^{10}$. Here, Nédélec finite elements ${ }^{11,12}$ are employed. Moreover, further difficulties arises from the fact that a Coulomb gauge condition, which is always satisfied in plane or axisymmetric situations, is not automatically fulfilled and has to be cared for to decouple the equations for the electromagnetic fields. In this work, a novel non-isoparametric version of Nédélec elements is presented working with trial functions with zero divergence such that the computed approximation fulfills a Coulomb gauge without any further requirements (see section 2).

There are several coupling mechanisms between the thermomechanical and the electromagnetic subsystem. On the one hand, the Lorentz force computed from the electromagnetic simulation serves as load term in the mechanical impulse balance. On the other hand, the conductivity distribution entering the electromagnetic simulation via the diffusivity is determined by the current position of the structure. Further, the electromotive intensity represents an additional coupling term. The most natural way to discretize the field equations in the context of their usual formulation is to employ a fixed Eulerian mesh for the electromagnetic field and to use a moving Lagrangian mesh for the mechanical structure. However, it has turned out that such an approach leads to serious problems in the data transfer between the fixed and the moving mesh. Particular, the computed Lorentz forces tend to oscillate and are not sufficiently accurate. To overcome this difficulty an arbitrary Lagrangian Eulerian (ALE) formulation for the electromagnetic field has additionally been developed (see section 4 ).

The paper is organized as follows: In section 2, the model relations governing the coupled multifield model are presented. The algorithmic formulation of the mechanical and of the electromagnetic subsystem in the context of the finite element method is described in section 3. Next, the incorporation of the coupling between the two subsystems is discussed in section 4 . The paper ends with some conclusions.

\section{COUPLED ELECTROMAGNETIC-MECHANICAL MODEL}

The multifield material model applied in this work is derived from a general continuum thermodynamic approach ${ }^{7,8}$ to the formulation of models for electromagnetic thermoelastic solids. For all structural problems of interest the frequencies of relevance (i.e., less than $10 \mathrm{MHz}$ ) correspond to electromagnetic wave lengths which are much larger than the structures of interest. Hence, the wave character of the electromagnetic fields is insignificant and can be neglected for such structural problems. This represents the so-called quasistatic approximation ${ }^{13}$. In this case, it is shown ${ }^{8}$ that Maxwell's relations together 
with Ohm's law and the Coulomb gauge condition ${ }^{14} \operatorname{div}_{\mathrm{s}} \boldsymbol{a}=0$ result in the weak forms

$$
\begin{aligned}
& \int_{R}^{*} \boldsymbol{a} \cdot \boldsymbol{a}_{*}+\left\{\zeta \boldsymbol{I}+\kappa_{\mathrm{EM}} \nabla_{\mathrm{S}} \boldsymbol{a}\right\} \cdot \nabla_{\mathrm{S}} \boldsymbol{a}_{*}=\int_{\partial R}\left\{\zeta \boldsymbol{I}+\kappa_{\mathrm{EM}} \nabla_{\mathrm{S}} \boldsymbol{a}\right\} \boldsymbol{n} \cdot \boldsymbol{a}_{*} \\
& \int_{R} \nabla_{\mathrm{S}} \chi \cdot \nabla_{\mathrm{S}} \chi_{*}=\int_{\partial R}\left(\nabla_{\mathrm{S}} \chi \cdot \boldsymbol{n}\right) \chi_{*}
\end{aligned}
$$

for all test fields $\boldsymbol{a}_{*}$ and $\chi_{*}$ with respect to a domain $R$ containing the workpiece, the tool coil, and a large area of air around the tool coil and the workpiece. As usual, the test fields vanish on those parts of $\partial R$ where $\boldsymbol{a}$ and $\chi$ are specified. Here, $\chi$ denotes the electric scalar potential, $\boldsymbol{a}$ the magnetic vector potential, connected to the flux density $\boldsymbol{b}$ via $\boldsymbol{b}=\operatorname{curl}_{\mathrm{S}} \boldsymbol{a}, \zeta:=\chi-\boldsymbol{a} \cdot \boldsymbol{v}$ a Euclidean frame-indifferent form of the scalar potential, $\boldsymbol{I}$ a second order unit tensor, $\stackrel{*}{\boldsymbol{a}}:=\partial \boldsymbol{a}+\left(\nabla_{\mathrm{s}} \boldsymbol{a}\right) \boldsymbol{v}+\left(\nabla_{\mathrm{s}} \boldsymbol{v}\right)^{\top} \boldsymbol{a}$ the objective time-derivative of $\boldsymbol{a}$, and $\kappa_{\mathrm{EM}}=\sigma_{\mathrm{EM}}^{-1} \mu_{\mathrm{EM}}^{-1}$ the magnetic diffusivity computed from the electric conductivity $\sigma_{\mathrm{EM}}$ and the permeability $\mu_{\mathrm{EM}}$. For the materials involved ferro-magnetic effects are not relevant and $\mu_{\mathrm{EM}}$ can be constantly set to the value of the permeability of the vacuum. The conductivity $\sigma_{\mathrm{EM}}$ equals zero outside the tool coil and the workpiece, resulting in an infinite diffusivity there. This means that the equilibrium distribution of the magnetic vector potential depending on its current values on the interface to the tool coil and the workpiece is instantaneously assumed in each time step. Finally, $\nabla_{\mathrm{s}}$ represents the usual nabla-operator in the spatial variables. Note that the Coulomb gauge condition, which effects a decoupling of the two equations in (1), does not automatically hold for threedimensional problems such that it has to be considered explicitly in a numerical solution scheme (see section 3). On the timescale $\tau_{\text {Exp }} \sim 10^{-4}$ s relevant to EMF the typical order of magnitude $\kappa_{\mathrm{EM}} \sim 10^{-1} \mathrm{~m}^{2} \mathrm{~s}^{-1}$ implies that magnetic diffusion will be important in the process since it takes place over lengthscales of $\sqrt{\kappa_{\mathrm{EM}} \tau_{\mathrm{Exp}}} \sim 10 \mathrm{~cm}$, which are significantly larger than the smallest dimension of interest (e.g., sheet metal thickness $\sim 1 \mathrm{~mm}$ ).

Turning next to the mechanical part of the coupled model, the weak momentum balance for the deformation field $\boldsymbol{\xi}$ is given by

$$
\int_{B_{\mathrm{r}}}\left(\varrho_{\mathrm{r}} \ddot{\boldsymbol{\xi}}-\boldsymbol{f}\right) \cdot \boldsymbol{\xi}_{*}+\boldsymbol{P} \cdot \nabla_{\mathrm{r}} \boldsymbol{\xi}_{*}=\int_{\partial B_{\mathrm{r}}}\left|\operatorname{cof}(\boldsymbol{F}) \boldsymbol{n}_{\mathrm{r}}\right| \boldsymbol{t}_{\mathrm{c}} \cdot \boldsymbol{\xi}_{*}
$$

with respect to the referential configuration $B_{\mathrm{r}} \subset R$ of the workpiece for all corresponding test fields $\boldsymbol{\xi}_{*}$ vanishing on those parts of the current boundary $\partial B_{\mathrm{c}}$ where $\boldsymbol{\xi}$ is specified. Here,

$$
\boldsymbol{f}=\operatorname{det}(\boldsymbol{F}) \boldsymbol{j} \times \boldsymbol{b}=\operatorname{det}(\boldsymbol{F}) \cdot \sigma_{\mathrm{EM}}\left(-\partial \boldsymbol{a}-\nabla_{\mathrm{s}} \chi+\boldsymbol{v} \times \boldsymbol{b}\right) \times \boldsymbol{b}
$$

represents the Lorentz (body) force (density), $\boldsymbol{P}$ the first Piola-Kirchhoff stress, $\boldsymbol{F}:=\nabla_{\mathrm{r}} \boldsymbol{\xi}$ the deformation gradient, $\boldsymbol{t}_{\mathrm{c}}$ the current boundary traction, and $\varrho_{\mathrm{r}}$ the referential mass density. Further, $\boldsymbol{j}$ denotes the current density which is related to the scalar and vector potential via Ohm's law and the definition of these potentials ${ }^{14}$. 
The mechanical model relations are completed by the specification of the material model. Here, attention is restricted to the metallic work-piece, which is modeled as a hyperelastic, viscoplastic material. For simplicity, the (mild) elastic and flow anisotropy of the metals of interest (e.g., Al) is neglected, as well as any kinematic hardening. More generally, e.g., for the case of deformation-induced anisotropic flow behavior ${ }^{15,16}$, this is of course no longer possible. Since the metal forming processes of interest are predominantly monotonic in nature, however, this last assumption is not unreasonable. In this case, the constitutive model is specified via the form of the referential free energy density $\psi_{\mathrm{r}}\left(\ln \boldsymbol{V}_{\mathrm{E}}, \epsilon_{\mathrm{PP}}\right)$ together with the evolution relations for the elastic left logarithmic stretch tensor $\ln \boldsymbol{V}_{\mathrm{E}}$ and accumulated equivalent inelastic deformation measure $\epsilon_{\mathrm{PP}}$. In the context of small elastic strain, the usual Hooke-based form

$$
\psi_{\mathrm{r}}\left(\ln \boldsymbol{V}_{\mathrm{E}}, \epsilon_{\mathrm{PP}}\right)=\frac{1}{2} \kappa_{\mathrm{r}}\left(\boldsymbol{I} \cdot \ln \boldsymbol{V}_{\mathrm{E}}\right)^{2}+\mu_{\mathrm{r}} \operatorname{dev}\left(\ln \boldsymbol{V}_{\mathrm{E}}\right) \cdot \operatorname{dev}\left(\ln \boldsymbol{V}_{\mathrm{E}}\right)+\psi_{\mathrm{P}}\left(\epsilon_{\mathrm{PP}}\right)
$$

is relevant. Here, $\operatorname{dev}\left(\ln \boldsymbol{V}_{\mathrm{E}}\right)$ denotes the deviatoric part of the left logarithmic stretch tensor. Another common assumption in the non-isothermal context in the case of metals is that of constant specific heat ${ }^{17}$. Here, $\kappa_{\mathrm{r}}$ represents the bulk modulus, $\mu_{\mathrm{r}}$ the shear modulus, and $\psi_{\mathrm{p}}\left(\epsilon_{\mathrm{PP}}\right)$ the contribution from energy storage due to isotropic hardening processes as usual. From (4), one obtains in particular the usual hyperelastic form

$$
\boldsymbol{K}=\partial_{\ln \boldsymbol{V}_{\mathrm{E}}} \psi_{\mathrm{r}}=3 \kappa_{\mathrm{r}} \operatorname{sph}\left(\ln \boldsymbol{V}_{\mathrm{E}}\right)+2 \mu_{\mathrm{r}} \operatorname{dev}\left(\ln \boldsymbol{V}_{\mathrm{E}}\right)
$$

for the Kirchhoff stress $\boldsymbol{K}=\boldsymbol{P} \boldsymbol{F}^{\top}$, where $\operatorname{sph}\left(\ln \boldsymbol{V}_{\mathrm{E}}\right)$ represents the sperical part of $\ln \boldsymbol{V}_{\mathrm{E}}$. As usual, $\psi_{p}\left(\epsilon_{\mathrm{PP}}\right)$ is estimated with the help of fits to the quasi-static yield curve for the materials of interest at room temperature.

Consider next the evolution of the internal variables and the inelastic behavior. In the metallic polycrystalline materials of interest at low-to-moderate homologous temperature, inelastic deformation processes are controlled predominantly by the activation of dislocation glide on glide systems ${ }^{18,19}$. Indeed, this seems to be the case even at high strain rates $^{20}$. Apparently, higher homologous temperature is required for other mechanics such as dislocation climb or even dynamic recrystallization to begin playing a role. Resistance to dislocation glide arising due to obstacles and other factors is related in the phenomenological context to hardening behavior. Quasi-static processes of this nature contributing to energy storage in the material result in the contribution

$$
-\varsigma_{\mathrm{p}}:=\psi_{\mathrm{r}, \epsilon_{\mathrm{p}}}
$$

to the effective quasi-static flow stress in the material. Such resistance to dislocation motion can be overcome by thermal fluctuation under the action of the local effective stress, represented in the current phenomenological context by $\sigma_{\mathrm{vM}}(\boldsymbol{K})+\varsigma_{\mathrm{P}}-\sigma_{\mathrm{F} 0}$, where $\sigma_{\mathrm{vM}}(\boldsymbol{K})$ represents the von Mises effective stress with respect to $\boldsymbol{K}$, and $\sigma_{\mathrm{F} 0}$ is the initial flow stress. On this basis,

$$
f_{\mathrm{P}}\left(\boldsymbol{K}, \varsigma_{\mathrm{P}}\right):=\sigma_{\mathrm{vM}}(\boldsymbol{K})+\varsigma_{\mathrm{P}}-\sigma_{\mathrm{F} 0}
$$


represents an activation measure or overstress in the current rate-dependent context. A power-law approximation of the more exact transition-state-based micromechanical relations for the kinetics of dislocation glide ${ }^{18,19}$ leads to the power-law form

$$
\phi\left(\epsilon_{\mathrm{p}}, \boldsymbol{D}, \boldsymbol{K}, \varsigma_{\mathrm{P}}\right)=\frac{\gamma_{\mathrm{p}}\left(\epsilon_{\mathrm{P}}, \boldsymbol{D}\right) \sigma_{\mathrm{P}}\left(\epsilon_{\mathrm{P}}, \boldsymbol{D}\right)}{m\left(\epsilon_{\mathrm{P}}, \boldsymbol{D}\right)+1}\left\langle\frac{f_{\mathrm{p}}\left(\boldsymbol{K}, \varsigma_{\mathrm{P}}\right)}{\sigma_{\mathrm{P}}\left(\epsilon_{\mathrm{P}}, \boldsymbol{D}\right)}\right\rangle^{m_{\mathrm{P}}\left(\epsilon_{\mathrm{p}}, \boldsymbol{D}\right)+1}
$$

upon which the evolution of the internal variables is based. Here, $\sigma_{\mathrm{F} 0}$ represents the initial flow stress, $\gamma_{\mathrm{p}}$ a characteristic strain-rate, $\sigma_{\mathrm{P}}$ the characteristic or effective activation stress magnitude, and $m$ the strain-rate exponent. Further, $\langle x\rangle:=\frac{1}{2}(x+|x|)$ represents the ramp function. As indicated, $\gamma_{\mathrm{P}}, \sigma_{\mathrm{P}}$ and $m_{\mathrm{P}}$ are in general functions of accumulated inelastic deformation and deformation rate. For simplicity, however, they will be treated as constants in the algorithmic formulation to follow. To indicate this, we write $\gamma_{0} \widehat{=} \gamma_{\mathrm{p}}$, $\sigma_{0} \widehat{=} \sigma_{\mathrm{P}}$, and $m_{0} \widehat{=} m_{\mathrm{P}}$. The form (8) determines the evolution relations

$$
\begin{aligned}
-\ln \boldsymbol{V}_{\mathrm{E}} & =\partial_{\boldsymbol{K}} \phi=\sqrt{\frac{3}{2}} \operatorname{sgn}(\operatorname{dev}(\boldsymbol{K})) \dot{\epsilon}_{\mathrm{P}} & & (\boldsymbol{K} \neq \mathbf{0}), \\
\dot{\epsilon}_{\mathrm{P}} & =\partial_{\mathrm{S}_{\mathrm{P}}} \phi=\gamma_{0}\left\langle\frac{f_{\mathrm{P}}}{\sigma_{0}}\right\rangle^{m_{0}} & & \left(f_{\mathrm{P}}>0\right),
\end{aligned}
$$

for the evolution of the internal variables, with

$$
\ln \boldsymbol{V}_{\mathrm{E}}:=\frac{1}{2} \ln \left(\boldsymbol{F} \overline{\boldsymbol{C}_{\mathrm{P}}^{-1}} \boldsymbol{F}^{\mathrm{\top}}\right)
$$

in terms of the inverse plastic right Cauchy-Green deformation $\boldsymbol{C}_{\mathrm{P}}^{-1}$. Above, $\operatorname{sgn}(\operatorname{dev}(\boldsymbol{K}))$ denotes the sign of the deviatoric part of the Kirchhoff stress.

As indicated, $\phi$ is differentiable in $\varsigma_{\mathrm{p}}$ everywhere except at $f_{\mathrm{p}}=0$, as well as in $\boldsymbol{K}$ everywhere except at $f_{\mathrm{p}}=0$ and at $\boldsymbol{K}=\mathbf{0}$. The corresponding subdifferentials exist everywhere. In the context of these forms for the evolution of the internal variables, the constraint $\gamma_{\mathrm{p}}\left(\epsilon_{\mathrm{p}}, \mathbf{0}\right)=0$ on the constitutive form of $\gamma_{\mathrm{p}}$ follows from the general thermodynamic analysis by Svendsen and Chanda ${ }^{8}$. In addition, Ohm's law together with the assumption that these evolution relations are independent of $\boldsymbol{\epsilon}$ implies $\sigma_{\mathrm{EM}} \geq 0$. Lastly, the restriction that $\gamma_{\mathrm{p}, \boldsymbol{D} \boldsymbol{D}}\left(\epsilon_{\mathrm{p}}, \mathbf{0}\right)$ be non-negative definite (i.e., for $f_{\mathrm{p}}>0$ ) follows from the thermodynamic analysis via the assumption that the non-equilibrium (i.e., dynamic) part of $\boldsymbol{K}$ is negligible. For details, the reader is referred to Svendsen and Chanda ${ }^{7,8}$.

\section{ALGORITHMIC FORMULATION}

In this section, an algorithmic formulation both for the mechanical and for the electromagnetic subsystem is presented. As discussed in the previous section, the difference in electromagnetic and mechanical timescales, together with the distinct nature of the respective fields involved (i.e., Eulerian in the electromagnetic case, Lagrangian in the mechanical large-deformation context) suggest that a staggered numerical solution procedure based on separate meshes for both field problems will be most efficient. 


\subsection{Algorithmic formulation of the mechanical model}

The algorithmic formulation of the mechanical model is carried out in the standard context of backward-Euler integration of the local evolution relations and their implicit solution via Newton-Raphson iteration in the context of the implicit function theorem ${ }^{21}$. Consistent linearization of the resulting algebraic system then facilitates the corresponding element formulation of the material model and its incorporation in the finite element solution of the dynamic initial-boundary-value problem.

To this end, the time interval $[0, d]$ of interest is split into $m$ subintervals $\left[0, t_{1}\right], \ldots$, $\left[t_{m-1}, t_{m}\right]$, such that $[0, d]=\bigcup_{i=0}^{m-1}\left[t_{i}, t_{i+1}\right]$ with $t_{0}=0, t_{i}<t_{i+1}$ and $t_{m}=d$. As usual, assume that the initial-boundary-value problem and internal variable evolution relations have been solved up to the discrete time $t=t_{n}$, such that $\boldsymbol{\xi}_{n}, \boldsymbol{a}_{n}$, and $\chi_{n}$, together with their spatial and temporal derivatives, as well as the corresponding internal variables, are all known in the corresponding region of Euclidean space at this time.

Since we are neglecting temperature effects here, the influence of the electromagnetic fields on the development of the mechanical fields is restricted to the Lorentz force (3). Equation (3) shows, that it depends on both electromagnetic and mechanical fields as well as their spatial and temporal derivatives. Since the electromagnetic fields vary on a timescale much smaller than that of the mechanical fields, a scaling analysis shows that the convective term $\boldsymbol{b} \times \boldsymbol{v}=\operatorname{curl}_{\mathrm{s}} \boldsymbol{a} \times \boldsymbol{v}$ is much smaller than the others and can be neglected. In this case, we work with the algorithmic approximation

$$
\boldsymbol{l}_{n+1} \approx \sigma_{\mathrm{EM}} \operatorname{curl}_{\mathrm{s}} \boldsymbol{a}_{n+1} \times\left(\partial \boldsymbol{a}_{n+1}+\nabla_{\mathrm{s}} \chi_{n+1}\right)
$$

for the current Lorentz force which is then "purely electromagnetic" in character. In particular, in the context of the staggered approach being pursued here, the purely electromagnetic terms $\operatorname{curl}_{\mathrm{s}} \boldsymbol{a}_{n+1}, \partial \boldsymbol{a}_{n+1}$ and $\nabla_{\mathrm{s}} \chi_{n+1}$ are treated in the mechanical part of the staggered algorithm as being known and fixed.

The next global step begins with the update of the boundary conditions to the next discrete time $t=t_{n+1}$ for the time interval $\left[t_{n}, t_{n+1}\right]$ of duration $t_{n+1, n}:=t_{n+1}-t_{n}$. Consider first the local algorithm at the Gauss-point level for the internal variables and the Kirchhoff stress $\boldsymbol{K}$. In the backward-Euler context, these are determined as usual as implicit functions of the current (unknown) deformation gradient $\boldsymbol{F}_{n+1}$ and (in the current rate-dependent context) time-step size $t_{n+1, n}$. In particular, the backward-Euler integration of (9) over $\left[t_{n}, t_{n+1}\right]$ and subsequent solution of the resulting non linear system of algebraic equations leads to the weak form ${ }^{9}$

$$
m_{n+1, n}\left(\boldsymbol{\xi}_{n+1}, \boldsymbol{\alpha}_{n+1}, \boldsymbol{\xi}_{*}\right)=0
$$

for all $\boldsymbol{\xi}_{*}$, of the momentum balance for the current unknown deformation field $\boldsymbol{\xi}_{n+1}$ satisfying the current boundary conditions. The parameter $\boldsymbol{\alpha}_{n+1}$ represents the algorithmic solution of the evolution equations for the internal variables as described before ${ }^{9}$. The 
particular form of the weak momentum balance above reads

$$
\begin{aligned}
m_{n+1, n}\left(\boldsymbol{\xi}_{n+1}, \boldsymbol{\alpha}_{n+1}, \boldsymbol{\xi}_{*}\right) & :=\int_{B_{\mathrm{r}}}\left\{\varrho_{\mathrm{r}} \boldsymbol{a}_{n+1, n}\left(\boldsymbol{\xi}_{n+1}\right)-\operatorname{det}\left(\nabla_{\mathrm{r}} \boldsymbol{\xi}_{n+1}\right) \boldsymbol{l}_{n+1}\right\} \cdot \boldsymbol{\xi}_{*} \\
& +\int_{B_{\mathrm{r}}} \boldsymbol{K}_{n}\left(\boldsymbol{\alpha}_{n+1}, \nabla_{\mathrm{r}} \boldsymbol{\xi}_{n+1}\right) \cdot \nabla_{n+1} \boldsymbol{\xi}_{*} \\
& -\int_{\partial B_{\mathrm{r}}}\left|\boldsymbol{c}_{\mathrm{r}}\left(\nabla_{\mathrm{r}} \boldsymbol{\xi}_{n+1}\right)\right| \boldsymbol{t}_{\mathrm{c} n+1} \cdot \boldsymbol{\xi}_{*}
\end{aligned}
$$

Again, in the context of the current staggered approach, the Lorentz force $\boldsymbol{l}_{n+1}$ is assumed known and given. The material acceleration ${ }^{1} \boldsymbol{a}_{n+1, n}\left(\boldsymbol{\xi}_{n+1}\right):=\boldsymbol{a}\left(\boldsymbol{\xi}_{n+1}, t_{n+1, n} ; \boldsymbol{\xi}_{n}, \dot{\boldsymbol{\xi}}_{n}, \ddot{\boldsymbol{\xi}}_{n}\right)$ is considered algorithmically a function of $\boldsymbol{\xi}_{n+1}, t_{n+1, n}$, and the state at the end $t=t_{n}$ of the last time step, in the context of, e.g., the Newmark algorithm. In addition, the notations $\nabla_{n+1} \boldsymbol{\xi}_{*}:=\left(\nabla_{\mathrm{r}} \boldsymbol{\xi}_{*}\right) \boldsymbol{F}_{n+1}^{-1}$ and that $\boldsymbol{c}_{\mathrm{r}}(\boldsymbol{F}):=\operatorname{cof}(\boldsymbol{F}) \boldsymbol{n}_{\mathrm{r}}$ have been introduced, with $\boldsymbol{n}_{\mathrm{r}}$ the outward unit normal to the boundary $\partial B_{\mathrm{r}}$ of $B_{\mathrm{r}}$.

As usual, the finite-element approximation to (13) in the Lagrangian context is based on the discretization $B \approx \bigcup_{e} B^{e}$ of $B$ into a finite number of elements $B^{1}, B^{2}, \ldots$ In terms of the corresponding finite-element approximation

$$
\boldsymbol{\xi}_{e}=\mathbf{H x}^{e}
$$

for the element deformation field $\boldsymbol{\xi}_{e}$ with the element shape function matrix $\mathbf{H}$ and the element nodal position vector $\mathbf{x}^{e}$, one obtains the element representation

$$
\boldsymbol{F}^{e}\left(\mathbf{x}^{e}\right):=\nabla_{\mathrm{r}}^{e} \boldsymbol{\xi}_{e}=\left(\nabla_{\mathrm{r}}^{e} \mathbf{H}\right)^{\mathrm{s}} \mathbf{x}^{e}
$$

for the deformation gradient, with $\left(\nabla_{\mathrm{r}}^{e} \mathbf{H}\right)^{\mathrm{s}} \mathbf{x}^{e}:=\nabla_{\mathrm{r}}^{e}\left(\mathbf{H} \mathbf{x}^{e}\right)$. In particular, these induce the discretized form

$$
m_{n+1, n}\left(\boldsymbol{\xi}_{n+1}, \boldsymbol{\alpha}_{n+1}, \boldsymbol{\xi}_{*}\right)=\sum_{e} \mathbf{f}_{n+1, n}^{e}\left(\mathbf{x}_{n+1}^{e}, \mathbf{e}_{n+1}^{e}\right) \cdot \mathbf{x}_{*}^{e}
$$

of the functional in (13), with

$$
\begin{aligned}
& \mathbf{f}_{n+1, n}^{e}\left(\mathbf{x}_{n+1}^{e}, \mathbf{e}_{n+1}^{e}\right):= \\
& \int_{B_{\mathrm{r}}^{e}} \mathbf{H}^{\top} \mathbf{H}\left\{\varrho_{0} \mathbf{a}_{n+1, n}^{e}\left(\mathbf{x}_{n+1}^{e}\right)-\operatorname{det}\left(\boldsymbol{F}^{e}\left(\mathbf{x}_{n+1}^{e}\right)\right) \ell_{n+1}^{e}\right\}+\left(\nabla_{n+1}^{e} \mathbf{H}\right)^{\mathrm{S \top}} \boldsymbol{K}_{n+1, n}^{e}\left(\mathbf{e}_{n+1}^{e}, \boldsymbol{F}^{e}\left(\mathbf{x}_{n+1}^{e}\right)\right) \\
& -\int_{\partial B_{\mathrm{r}}^{e}}\left|\boldsymbol{c}_{\mathrm{r}}\left(\boldsymbol{F}^{e}\left(\mathbf{x}_{n+1}^{e}\right)\right)\right| \mathbf{H}^{\top} \boldsymbol{t}_{\mathrm{c} n+1}^{e}
\end{aligned}
$$

\footnotetext{
${ }^{1}$ Not to be confused with the vector potential $\boldsymbol{a}$.
} 
with $\nabla_{n+1}^{e} \mathbf{H}:=\left(\nabla_{\mathrm{r}}^{e} \mathbf{H}\right) \boldsymbol{F}_{n+1}^{-1}$ and $\boldsymbol{l}^{e}=\mathbf{H} \boldsymbol{\ell}^{e}$. Further, $\boldsymbol{\ell}^{e}$ denotes the nodal force vector. With the help of the connectivity relations

$$
\mathbf{x}^{e}=\mathbf{I}_{\mathbf{x}}^{e} \mathbf{x}^{s}
$$

between the element and structural nodal positions, (16) reduces to

$$
m_{n+1, n}\left(\boldsymbol{\xi}_{n+1}, \boldsymbol{\alpha}_{n+1}, \boldsymbol{\xi}_{*}\right)=\mathbf{f}_{n+1, n}^{s}\left(\mathbf{x}_{n+1}^{s}, \mathbf{e}_{n+1}^{s}\right) \cdot \mathbf{x}_{*}^{s}
$$

with

$$
\mathbf{f}_{n+1, n}^{s}\left(\mathbf{x}_{n+1}^{s}, \mathbf{e}_{n+1}^{s}\right):=\sum_{e} \mathbf{I}_{\mathbf{x}}^{e \top} \mathbf{f}_{n+1, n}^{e}\left(\mathbf{I}_{\mathbf{x}}^{e} \mathbf{x}_{n+1}^{s}, \mathbf{e}_{n+1}^{e}\right)
$$

Since $\mathbf{x}_{*}^{s}$ is arbitrary, (12) reduces to the discrete form

$$
\mathbf{f}_{n+1, n}^{s}\left(\mathbf{x}_{n+1}^{s}, \mathbf{e}_{n+1}^{s}\right)=\mathbf{0}
$$

in terms of $\mathbf{f}_{n+1, n}^{s}$. For its solution via Newton Raphson iteration the algorithmic derivative

$$
\partial_{\mathbf{x}_{n+1}^{s}}^{\mathrm{a}} \mathbf{f}_{n+1, n}^{s}=\sum_{e} \mathbf{I}_{\mathbf{x}}^{e \boldsymbol{\top}}\left(\partial_{\mathbf{x}_{n+1}^{e}}^{\mathrm{a}} \mathbf{f}_{n+1, n}^{e}\right) \mathbf{I}_{\mathbf{x}}^{e}
$$

at fixed $\boldsymbol{\ell}_{n+1}^{s}$ is required. For its determination, the reader is referred to the literature ${ }^{9}$.

\subsection{Divergence-free discretization of the electromagnetic system}

Starting point of the finite element discretization of the electromagnetic field relations is their weak form (1). The right hand side of these equations represents the flux over the specified area $R$, which may be chosen as a large box containing the tool coil, the workpiece, and a large area of air surrounding them. If $R$ is chosen large enough, the approximation $\boldsymbol{a}=\mathbf{0}$ on $\partial R$ is well founded due to the asymptotic decay of the vector potential of a dipole field like $O\left(|x|^{-2}\right),|x| \rightarrow \infty$. The values of $\chi$ can be specified by the electric current flowing through $\partial R$ via Neumann conditions. Alternatively, the boundary values of $\chi$ can be determined by measured electric potentials on $\partial R$ if those are available, leading to Dirichlet data for the corresponding boundary value problem. As in the mechanical case the time interval $[0, d]$ of interest is split into $m$ subintervals $\left[0, t_{1}\right], \ldots,\left[t_{m-1}, t_{m}\right]$, such that $[0, d]=\bigcup_{i=0}^{m-1}\left[t_{i}, t_{i+1}\right]$ with $t_{0}=0, t_{i}<t_{i+1}$ and $t_{m}=d$. Again, we assume that discrete values $\boldsymbol{a}_{n}$ of the vector potential and of its time derivative have been computed up to a discrete time $t=t_{n}$. According to (1), there is no explicit dependence of past values of the electromagnetic scalar potential $\chi$.

The discretization of $\chi$ at a certain instant is based on a similar type of spatial discretization as chosen for the deformation field. The degrees of freedom are represented by (in contrast to the deformation field) one single scalar value at each node of the discretization $R \approx \bigcup_{e} R^{e}$ of $R$ into a finite number of elements. Each $R^{e}$ is considered to be the image of a reference element under transformations from an isoparametric family. In the following, we focus on hexahedra element, although other geometries are also possible. 
For convenience, the discretization of the vector potential shall be based on the same decomposition $R \approx \bigcup_{e} R^{e}$ as used for the computation of the scalar potential. However, a standard approach based on finite elements that enforce continuity leads here to a poor approximation of the jumps of the normal component of the electromagnetic field at material interfaces. There are several methods to cope with this difficulty, including penalty or least square methods ${ }^{10}$. In this work, Nédélec elements ${ }^{11,12}$ of lowest order are applied. They imitate the regularity of the electromagnetic field. Instead of values in the vertices of the cells of the finite elements discretization, integral mean values over the edges represent the degrees of freedom of these elements. For higher order Nédélec elements, also certain momenta over edges, faces and the whole cells are considered as degrees of freedom ${ }^{11,12}$, which improves the local approximation to sufficiently smooth functions and may increase the convergence rate of the finite element method. Nédélec elements have also been chosen by Schinnerl and Schöberl ${ }^{22}$ to simulate three-dimensional coupled electromagnetic mechanical systems, where emphasis is laid on a fast solution of the coupled system via a multigrid solver. However, these results do not apply to EMF since the mechanical system is restricted to linear elasticity.

As mentioned before, the field relations (1) only assume the stated decoupled form if a Coulomb gauge is provided. This could be done by explicitly demanding $\operatorname{div}_{\mathrm{s}} \boldsymbol{a}=0$ and solving the resulting saddle point problem. Here, we present a novel approach that is numerically less expensive: By choosing a non-isoparametric setting, it is possible to work with divergence-free test and trial functions in the context of Nédélec elements. Hence, any solution to the Galerkin discretization of (1) automatically fulfills the Coulomb gauge condition and is thus a solution of the electromagnetic field problem. More precise, to construct a discretization of the vector potential equation no transformation on a referential cube is performed, but in each element of the discretization $R \approx \bigcup_{e} R^{e}$ of $R$ a local basis of the test and trial space is constructed as follows: To each edge $\Gamma_{i}$ of a hexahedron $R^{e}, 1 \leq i \leq 12$, a basis function $b_{k}$ of the form

$$
b_{k}(x)=\left(\begin{array}{lllllll}
a_{11}^{(k)} & + & a_{12}^{(k)} x_{2} & + & a_{13}^{(k)} x_{3} & + & a_{14}^{(k)} x_{2} x_{3} \\
a_{21}^{(k)} x_{1} & + & a_{22}^{(k)} & + & a_{23}^{(k)} x_{3} & + & a_{24}^{(k)} x_{1} x_{3} \\
a_{31}^{(k)} x_{1} & + & a_{32}^{(k)} x_{2} & + & a_{33}^{(k)} & + & a_{34}^{(k)} x_{1} x_{2}
\end{array}\right)
$$

is assigned with

$$
\int_{\Gamma_{i}} b_{k} \cdot t_{i}=\delta_{k i},
$$

where $t_{i}$ represents a tangential vector to $\Gamma_{i}$ of unit length and $\delta_{k i}$ is defined by $\delta_{i i}=1$, $1 \leq i \leq 12$, and $\delta_{k i}=0$ for $k \neq i$ respectively. Algorithmically, the determination of the $b_{k}$ leads to the solution of 12 systems, each of which consists of 12 linear equations, in any element $R^{e}$ of the finite-element discretization. Particularly, a two point Gauss quadrature already yields exact values on the left hand side of (24). The tangential vectors $t_{i}$ can easily be computed from the current positions of the vertices. The necessary numerical 
efforts remain acceptably small since all 12 systems to be solved in a certain element possess the same system matrix and, e.g., MATLAB, solves 100,000 sets of 12 systems of this type within a CPU time of 5.36 s on a 2394 Mhz Opteron machine.

One easily deduces that all $b_{k}$ are divergence-free and such is the local approximation to the electromagnetic field being a linear combination of these basis functions. The method presented here deviates from the usual employment of Nédélec elements where a local basis obeying the above approach (23) is only constructed for a referential cube and then transferred by a family of isoparametric transformations on the physical elements such that integrals over the edges of the form (24) remain invariant. However, the local test and trial spaces obtained from this process are no longer divergence-free such that the Coulomb gauge condition is not generally satisfied. Hence, the weak forms (1) for $\boldsymbol{a}$ and $\chi$ to be discretized contain further coupling terms. Another drawback of an isoparametric family of Nédélec elements results from additional approximations that are usually undertaken in the practical implementation of the method: Certain second order terms that are dropped may become significantly large when the shape of the elements deviates too much from a parallelepiped, which may happen, if large deformations occur in the ALE context.

With the shape function matrix $\mathbf{N} \in \mathbb{R}^{12 \times 12}$ at hand constructed in the usual fashion from the basis functions $b_{k}$, one obtains

$$
a_{e}=\mathrm{Na}^{e}
$$

for the vector potential field at the element level $\boldsymbol{a}_{e}$. Here, $\mathbf{a}^{e} \in \mathbb{R}^{12}$ represents the vector of integral means

$$
\mathbf{a}_{i}^{e}=\int_{\Gamma_{i}} \boldsymbol{a} \cdot t_{i}, \quad i=1, \ldots, 12,
$$

over all edges $\Gamma_{i}$ of $R^{e}$. This implies

$$
\nabla_{\mathrm{s}}^{e} \boldsymbol{a}_{e}=\left(\nabla_{\mathrm{s}}^{e} \mathbf{N}\right)^{\mathrm{S}} \mathbf{a}^{e}
$$

for the corresponding gradient. On this basis, one obtains the spatially-discretized form

$$
\sum_{e}\left(\mathbf{A}^{e} \mathbf{a}^{e}+\mathbf{B}^{e} \dot{\mathbf{a}}^{e}\right) \cdot \mathbf{a}_{*}^{e}=\sum_{e} \mathbf{c}^{e} \cdot \mathbf{a}_{*}^{e}
$$

of (1) with the local contributions

$$
\mathbf{A}^{e}:=\int_{R^{e}}\left(\nabla_{\mathrm{s}}^{e} \mathbf{N}\right)^{\mathrm{ST}}\left(\nabla_{\mathrm{s}}^{e} \mathbf{N}\right)^{\mathrm{s}}, \quad \mathbf{B}^{e}:=\kappa_{\mathrm{EM}}^{-1} \int_{R^{e} \cap W} \mathbf{N}^{\top} \mathbf{N}, \quad \mathbf{c}^{e}:=\int_{R^{e} \cap W} \mathbf{N}^{\top} \nabla_{\mathrm{s}} \chi .
$$

The entries of the source vector $\mathbf{c}^{e}$ are computed from the solution $\chi$ of the electro-static equation. For simplicity, it has not been indicated in the notation that only a finiteelement approximation to $\chi$ is available. Using the connectivity relation

$$
\mathbf{a}^{e}=\mathbf{I}_{\mathbf{a}}^{e} \mathbf{a}^{s}
$$


between the element and structural integral mean values over edges of the vector potential, the arbitrariness of $\mathbf{a}_{*}^{e}$ leads to the structural form

$$
\mathbf{A}^{s} \mathbf{a}^{s}+\mathbf{B}^{s} \dot{\mathbf{a}}^{s}=\mathbf{c}^{s}
$$

of (28), with

$$
\mathbf{A}^{s}=\sum_{e} \mathbf{I}_{\mathbf{a}}^{e \top} \mathbf{A}^{e} \mathbf{I}_{\mathbf{a}}^{e}, \quad \mathbf{B}^{s}=\sum_{e} \mathbf{I}_{\mathbf{a}}^{e \top} \mathbf{B}^{e} \mathbf{I}_{\mathbf{a}}^{e}, \quad \mathbf{c}^{s}=\sum_{e} \mathbf{I}_{\mathbf{a}}^{e \top} \mathbf{c}^{e}
$$

Integration of (31) via the generalized trapezoidal rule over the interval $\left[t_{n}, t_{n+1}\right]$ yields the system

$$
\left[\begin{array}{cc}
\mathbf{A}^{s} & \mathbf{B}_{n+1}^{s} \\
\mathbf{I} & -\alpha t_{n+1, n} \mathbf{I}
\end{array}\right]\left[\begin{array}{c}
\mathbf{a}_{n+1}^{s} \\
\dot{\mathbf{a}}_{n+1}^{s}
\end{array}\right]=\left[\begin{array}{c}
\mathbf{c}_{n+1}^{s} \\
\mathbf{a}_{n}^{s}+(1-\alpha) t_{n+1, n} \dot{\mathbf{a}}_{n}^{s}
\end{array}\right]
$$

to solve for $\mathbf{a}_{n+1}^{s}$ and $\dot{\mathbf{a}}_{n+1}^{s}$, in the context of the current staggered approach with fixed $\mathbf{x}_{n+1}^{s}$. In contrast to $(21)$ for $\mathbf{x}_{n+1}^{s}$, note that this last relation can be solved explicitly for these quantities. Here, the parameter $0 \leq \alpha \leq 1$ controls the amount of artificial damping exerted on the discrete system ${ }^{23}$.

Equation (31) reduces to a time independent linear equation for those degrees of freedom that lie outside the tool coil or the work-piece due to $\kappa_{\mathrm{EM}}^{-1}=0$ there. These degrees of freedom depend only indirectly on the time by their coupling to those degrees of freedom lying in areas with $\kappa_{\mathrm{EM}}^{-1}>0$ (i.e., the tool coil or the work-piece). As in the mechanical case, all spatially-discretized integrals are evaluated via Gauss quadrature.

If we are interested in values of $\boldsymbol{a}$ in a particular spatial point, a certain postprocessing is required: First that element $R_{\overline{\mathrm{e}}}$ has to be identified that contains the point of interest. Then $\mathbf{N a}^{\bar{e}}$ has to be evaluated in this particular point, yielding the sought approximation.

\subsection{Simplifications in axisymmetric situations}

In an axisymmetric context, the vector potential always points in azimuthal direction and is thus perpendicular to all material interfaces. Hence, $\boldsymbol{a}$ is continuous and a standard finite element approach with 4-node elements leads to a sufficiently accurate approximation. In some situations, real forming devices can be approximated as being axisymmetric $^{9}$ as e.g. in the case displayed in Figure 1: Consider e.g. a coil $C_{\mathrm{c}}$ that consists of $n$ windings. Each winding can be approximated by a torus of the same cross section. The resulting $n$ tori are cut in the $(r, z)$-plane at $\varphi=0$. In order to simulate that each torus is in fact the arm of a spiral, the cross sections at $\varphi=0$ and $\varphi=2 \pi$ are treated electromagnetically as being continuous with respect to $\chi$. Continuity of potential then implies that $U$ at $\varphi=0$ (except in the first torus) is determined by that in the preceding torus at $\varphi=2 \pi$. Let $W_{\text {ck }}$ denote the current configuration of the $k^{\text {th }}$ torus, and $U_{k}$ the potential of $W_{\mathrm{ck}}$ at $\varphi=0$. Under these assumptions, the restriction $\chi_{k}$ of $\chi=\chi(r, \varphi, z)$ to $W_{\mathrm{ck}}$ is given $\mathrm{by}^{9}$

$$
\chi_{k}(r, \varphi, z)=U_{k}+\Delta U_{k} \frac{\varphi}{2 \pi}
$$


with $\Delta U_{k}=U_{k+1}-U_{k}$ The potential differences $\Delta U_{k}$ can be obtained from the measured total current $I=I(t)$, which is equal in all tori $W_{\mathrm{ck}}, k=1, \ldots, n$, since they are connected in series. This leads e.g., in the case of a coil with rectangular cross section, to

$$
\Delta U_{k}=-2 \pi\left(h \ln \frac{b_{k}}{a_{k}}\right)^{-1}\left\{\sigma_{\mathrm{EM}}^{-1} I+\int_{A_{\mathrm{c} k}} \partial \boldsymbol{a}_{k} \cdot \boldsymbol{n}_{k}\right\},
$$

where $h$ is the height (in $z$-direction) of each winding, $a_{k}$ the inner, and $b_{k}$ the outer radius of the $k^{\text {th }}$ winding. On this basis the integro-differential equation

$$
\partial \boldsymbol{a}_{k}-\kappa_{\mathrm{EM}} \nabla_{\mathrm{S}}^{2} \boldsymbol{a}_{k}+\frac{\Delta U_{k}}{2 \pi r} \boldsymbol{e}_{\varphi}=\mathbf{0}
$$

follows for the restriction $\boldsymbol{a}_{k}$ of $\boldsymbol{a}$ to $W_{\mathrm{ck}}$, with $\Delta U_{k}$ given by (35). Globally, this leads to

$$
\begin{aligned}
& \int_{R} \partial \boldsymbol{a} \cdot \boldsymbol{a}_{*}+\int_{R} \kappa_{\mathrm{EM}} \nabla_{\mathrm{S}} \boldsymbol{a} \cdot \nabla_{\mathrm{S}} \boldsymbol{a}_{*}+\int_{S_{c}} \operatorname{curl}_{\mathrm{S}} \boldsymbol{a} \times \boldsymbol{v} \cdot \boldsymbol{a}_{*} \\
& =\sum_{k=1}^{n} \int_{W_{\mathrm{ck}}} c_{k}\left\{\sigma_{\mathrm{EM}}^{-1} I+\int_{A_{\mathrm{ck}}} \partial \boldsymbol{a}_{k} \cdot \boldsymbol{n}_{k}\right\} \boldsymbol{e}_{\boldsymbol{\varphi}} \cdot \boldsymbol{a}_{*}
\end{aligned}
$$

for all test fields $\boldsymbol{a}_{*}$ in this particular situation. The algorithmic solution of this integrodifferential equation contains the difficulty that all degrees of freedom located in the same winding of the tool coil are coupled via the integral expression in (37). This corrupts both the symmetry and the sparse structure of the system matrix typical of the finite element method. However, sufficiently effective algorithmic formulations that are based on a standard isoparametric 4-node finite element approaches are possible ${ }^{9}$.

\section{COUPLING STRATEGIES}

In the above formulation the mechanical field is given in a Lagrangian formulation, while the electromagnetic field is given in an Eulerian formulation. The most natural discretization of the field equations leads to a moving mesh for the mechanical system, representing its current configuration and a fixed Eulerian mesh for the electromagnetic field. This approach is discussed in the first subsection and computational results are presented. An arbitrary Lagrangian Eulerian formulation for the electromagnetic system is presented in the second subsection.

The coupling between the two subsystems may in both cases be realized explicitly or implicitly. In an explicit coupling scheme the electromagnetic field of the $(n+1)^{\text {th }}$ time step is computed from the position of the structure in the $n^{\text {th }}$ time step and the position of the structure in the $(n+1)^{\text {th }}$ time step is then computed according to this field distribution. Hence, in any time step the electromagnetic and the deformation field are only computed once. In an implicit scheme, however, the electromagnetic field is several times recalculated in each time step according to the position of the altered structure and 
the structure is altered several times according to the changed electromagnetic field. The latter method is more stable and allows for larger time steps. However, if the numbers of unknowns is large an explicit method may be more efficient.

\subsection{Eulerian formulation of the electromagnetic system}

Recently, a coupled simulation has been presented ${ }^{9}$ where the discretization of the electromagnetic subsystem was based on an Eulerian formulation of the discrete system. This means that the Lagrangian mesh for the mechanical structure has been moved over a fixed Eulerian mesh for the electromagnetic field. In Figure 2 the particular choice of meshes is demonstrated. However, there are problems inherent to this approach since the
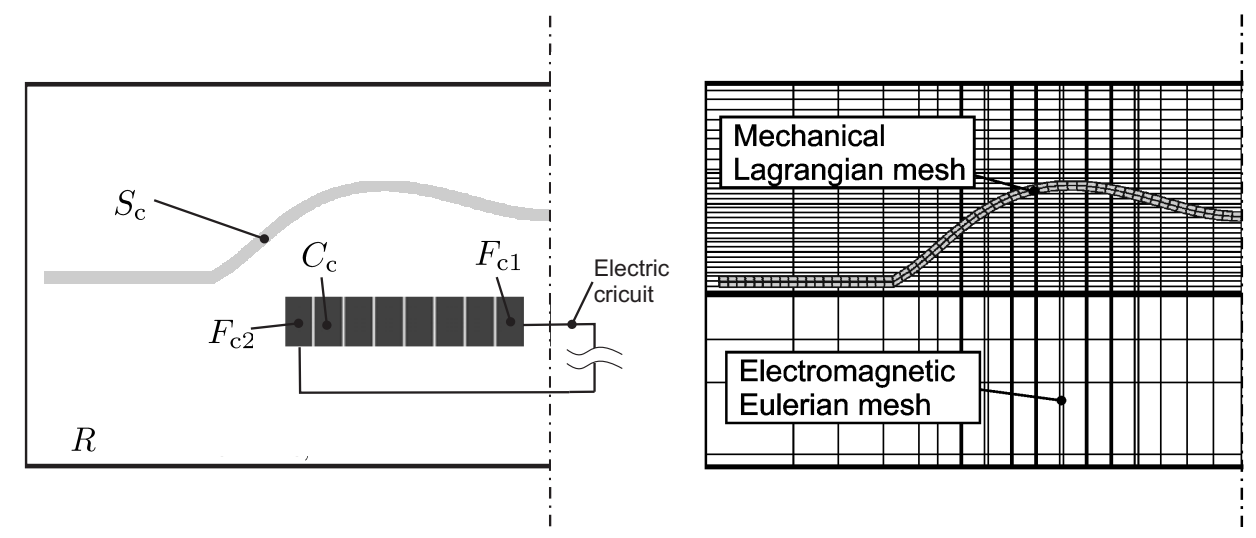

Figure 2: Meshing of the coupled system in case of a Lagrange-Euler-formulation.

character of the electromagnetic field equation in a certain point of the electromagnetic mesh changes from one instant to another when the structure moves over it: As long as it is not covered by the mechanical structure, the field equations are elliptic (instantaneous assumption of the equilibrium field) and they become parabolic (diffusion process) as soon as the point is covered by the structure. This leads to a sudden change in the local discretizations since a contribution to the mass matrix arises as soon as a point is covered by the structure and it disappears when it is uncovered again (see Figure 4). Particularly, for those points of the electromagnetic mesh covered by the mechanical structure values of the last time step are relevant, while the values in the other points do not depend explicitly on those of the preceding time step. It has turned out that this change of the discretization in a certain point causes oscillations in the time derivative $\partial \boldsymbol{a}$ of the vector potential and, thus, in the Lorentz force via the contribution $\sigma_{\mathrm{EM}} \partial \boldsymbol{a} \times \operatorname{curl}_{\mathrm{S}} \boldsymbol{a}$. These oscillations can be moderated by a very fine discretization of that part of the electromagnetic mesh lying in the interface region of the structure and the surrounding air. To avoid an inadequate fine discretization of the whole structure, adaptive techniques are necessary for a sufficiently fine resolution of the interface region. However, averaged quantities are quite good approximated with this approach, even with relatively coarse 
discretizations. Figure 3 displays the forming stages and the convergence behavior of the deformation field. Particularly, the above mentioned oscillations are smoothed out by the
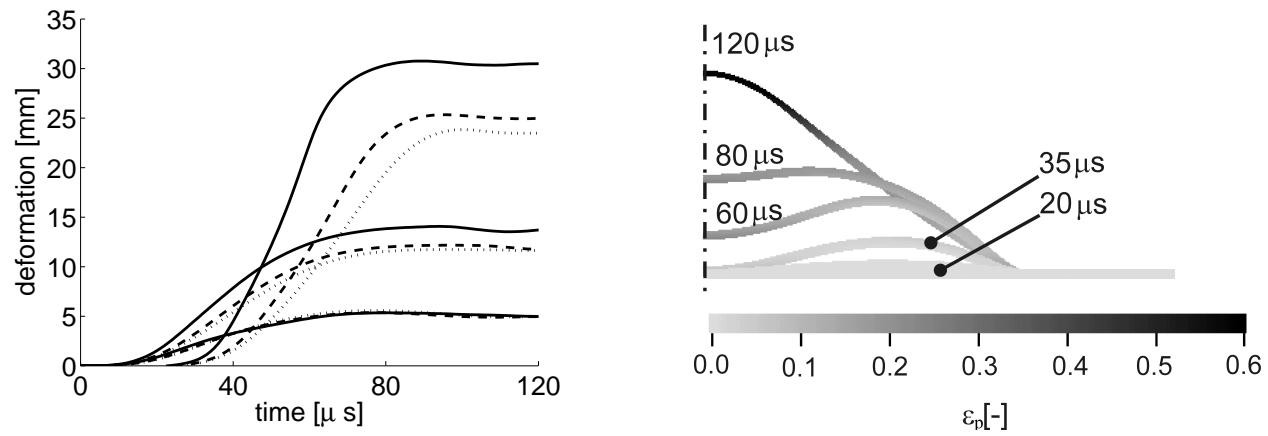

Figure 3: Forming stages (right) and convergence behavior (left) of the vertical displacement at three specified radii for the simulation of a free forming process as displayed in Figure 1. The workpiece (a sheet metal disk) is discretized by 2 (solid lines), 4 (dashed lines) and 8 (dotted lines) elements over its thickness. The discretization of the magnetic mesh is always maintained.

integration of Lorentz forces and due to the time stepping algorithm.

In the example presented above, the free forming of the aluminum alloy AC120 with the device displayed in Figure 1 has been considered. The simulations have been carried out with the simplifications for axisymmetric situations described in section 3.3. To apply the algorithmic formulation derived in section 3, the isotropic hardening behavior of the material has to be specified. As a first approximation, quasi-static uniaxial test data ${ }^{24}$ were used for this purpose. Identification of the semi-empirical form

$$
\psi_{\mathrm{P}}\left(\epsilon_{\mathrm{P}}\right)=c_{1}\left(\epsilon_{\mathrm{P}}+c_{2}\right)^{c_{3}}+c_{4} \ln \left(1+c_{5} \epsilon_{\mathrm{p}}\right)
$$

of the dependence of the inelastic part $\psi_{\mathrm{p}}$ of the free energy density on $\epsilon_{\mathrm{p}}$ related to energy storage in the material due to isotropic hardening based on these data yields $\sigma_{\mathrm{F} 0}=116.0$ $\mathrm{MPa}, c_{1}=-12.39 \mathrm{MPa}, c_{2}=0.001, c_{3}=0.0697, c_{4}=80.31 \mathrm{MPa}$ and $c_{5}=36.59$. In particular, this form determines the quasi-static contribution $\sigma_{\mathrm{Y}}=-\varsigma_{\mathrm{P}}$ to the flow stress from (6). Further, values of $\sigma_{0}=90 \mathrm{MPa}, \gamma_{0}=10^{4} \mathrm{~s}^{-1}$ and $m_{0}=5$ for aluminum relevant for strain-rates of $\geq 10^{3} \mathrm{~s}^{-1}$ were taken from the literature ${ }^{25}$. Lastly, the elastic behavior of AC120 is characterized by the values $\lambda=39404 \mathrm{MPa}$ and $\mu=26269 \mathrm{MPa}$ for the Lamé constants at room temperature.

\subsection{ALE-formulation of the electromagnetic field}

If a good approximation to the forces is required an ALE-based method is more promising. Here, the electromagnetic mesh is adapted to the moving structure such that always the same elements are covered by the moving mechanical structure (see Figure 4). Consequently, the character of the discretization in a particular element does never change, 

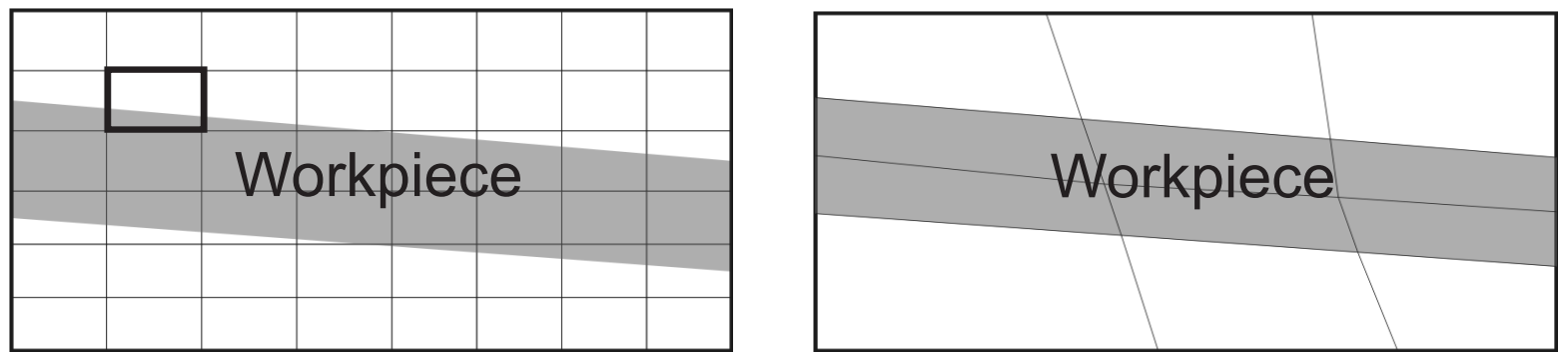

Figure 4: Comparison of the data transfer for a Lagrange-Euler-formulation (left hand side) and a Lagrange-ALE-approach (right hand side).

which avoids those jumps of $\partial \boldsymbol{a}$ that are typical of a Lagrange-Euler formulation. Meshes constructed by this algorithm are displayed in Figure 5 and 6 . While the simulation displayed in Figure 5 represents the same realistic forming process that was considered in subsection 4.1, the simulation displayed in Figure 6 is still of academic character. The incorporation of realistic three-dimensional geometries represents work in progress.

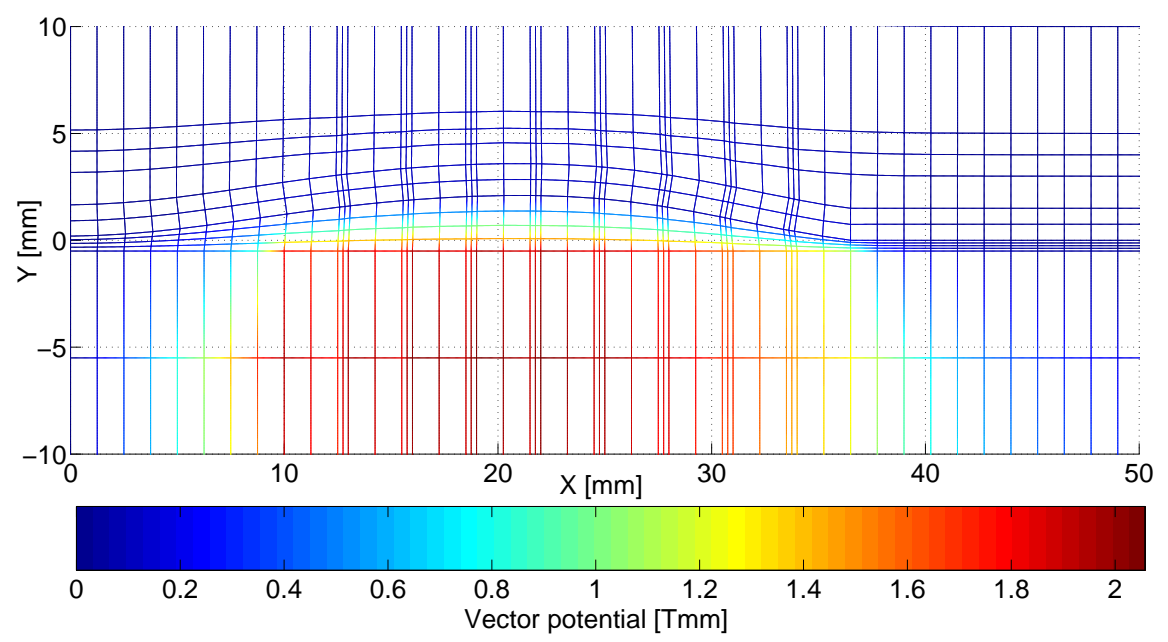

Figure 5: Mesh adaption in an ALE formulation (two-dimensional case). The same forming process as in the afore mentioned simulation based on an Eulerian formulation has been considered.

The movement of the electromagnetic mesh is arbitrary in the sense that the position of the discretizing mesh is not determined by requirements of the electromagnetic field equations themselves, but by accompanying conditions. To obtain a high quality mesh for the electromagnetic system with hexahedra elements that deviate as little as possible from the shape of a parallelepiped and that matches the mesh for the mechanical structure, the following algorithm is applied ${ }^{2}$ : Before a new time step is started each component

\footnotetext{
${ }^{2}$ Here, the three-dimensional case is described. The axisymmetric situation is treated analogously.
} 


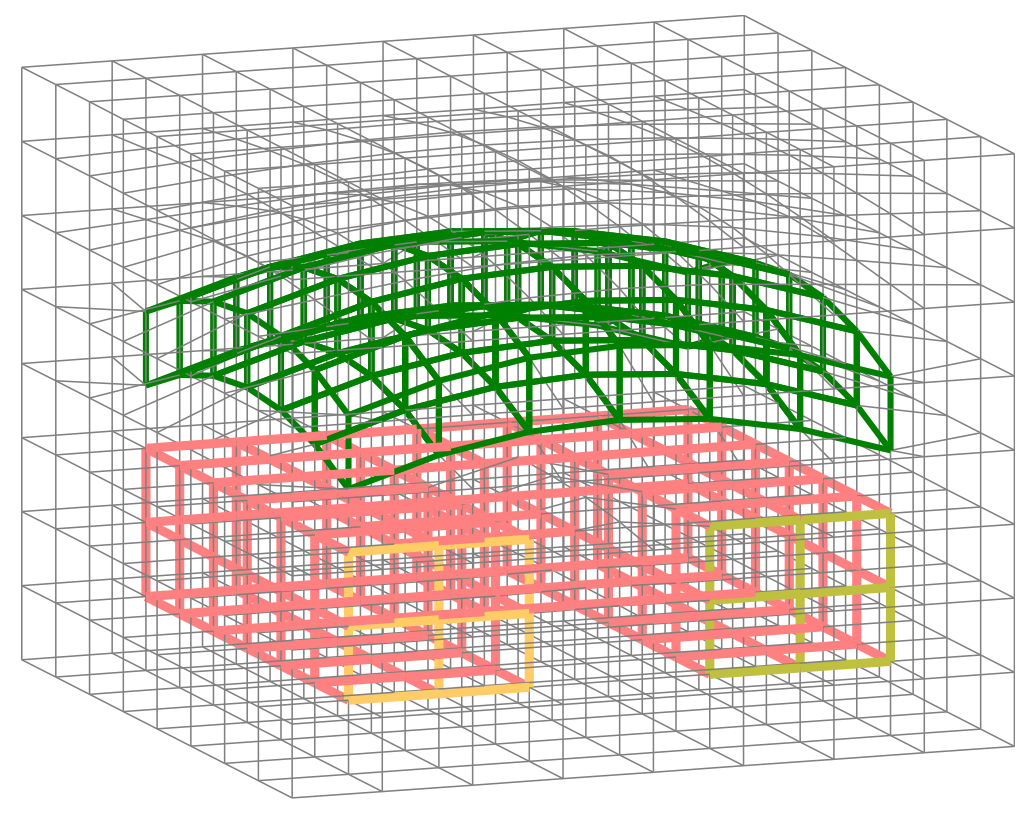

Figure 6: Mesh adaption in an ALE formulation (three-dimensional case).

of the deformation increment $d \boldsymbol{\xi}=\boldsymbol{\xi}_{n+1}-\boldsymbol{\xi}_{n}$ is considered as the boundary value of a one-dimensional Dirichlet problem

$$
\frac{\partial^{2} u_{k}}{\partial x_{1}^{2}}+\frac{\partial^{2} u_{k}}{\partial x_{2}^{2}}+\frac{\partial^{2} u_{k}}{\partial x_{3}^{2}}=0, \quad k=1, \ldots, 3,
$$

in the air around tool coil and workpiece (i.e., in those parts of $R$ that are outside the workpiece and outside the tool coil) with boundary values $u_{k}=d \xi_{k}, k=1, . ., 3$, on the interface between workpiece and air as well as $u_{k}=0$ on the interface between tool coil and air and on the outer boundary $\partial R$ (i.e., the mesh nodes are held fixed there). The solution of these problems (all possessing the same stiffness matrix) is added to the current positions of the electromagnetic mesh in the air-region around tool coil and workpiece to obtain a mesh for the next time step.

To get an impression of the quality of the arising meshes, one can consider the transformation from the old to the new mesh as an elastic deformation with no transversal contraction and with elasticity module 1 . This deformation is conducted by the boundary values on the interface to the tool coil, on the workpiece, and on the outer boundary since no forces are assumed to be present. In contrast to remeshing strategies, this approach preserves the combinatorial structure of the mesh, which allows an effective solution of the arising huge systems of linear equations by multigrid techniques.

The discrete field relations of the electromagnetic mesh have to be reformulated such 
that the movement of the mesh is correctly considered. Surprisingly, the resulting equations simplify. Instead of working with the partial time derivative $\partial \boldsymbol{a}$, it is convenient in this case to employ the material time derivative $\dot{\boldsymbol{a}}=\partial \boldsymbol{a}+\left(\nabla_{\mathrm{s}} \boldsymbol{a}\right) \boldsymbol{v}$ since its discretization is a function of the vertices of the moving mesh inside the mechanical structure rather than of spatial points. Thus, no interpolation is necessary to link past data to current data. Inside the fixed tool coil $\dot{\boldsymbol{a}}=\partial \boldsymbol{a}$ applies and in the air surrounding the tool coil and the workpiece the field assumes an equilibrium position instantaneously, which is explicitly neither depending on values of $\partial \boldsymbol{a}$ nor on values of $\boldsymbol{a}$ from a preceding time step. The weak form for the electromagnetic problem - still under the assumption that a Coulomb gauge is provided for - then takes the form

$$
\begin{aligned}
& \int_{R} \kappa_{\mathrm{EM}}^{-1}\left\{\dot{\boldsymbol{a}}+\nabla_{\mathrm{s}} \chi-\left(\nabla_{\mathrm{s}} \boldsymbol{a}\right)^{\top} \boldsymbol{v}\right\} \cdot \boldsymbol{a}_{*}+\nabla_{\mathrm{S}} \boldsymbol{a} \cdot \nabla_{\mathrm{S}} \boldsymbol{a}_{*}=\int_{\partial R}\left\{\zeta \boldsymbol{I}+\kappa_{\mathrm{EM}} \nabla_{\mathrm{s}} \boldsymbol{a}\right\} \boldsymbol{n} \cdot \boldsymbol{a}_{*}, \\
& \int_{R} \nabla_{\mathrm{s}} \chi \cdot \nabla_{\mathrm{S}} \chi_{*}=\int_{\partial R}\left(\nabla_{\mathrm{s}} \chi \cdot \boldsymbol{n}\right) \chi_{*} .
\end{aligned}
$$

Figure 7 shows results of a three-dimensional simulation carried out with this method.

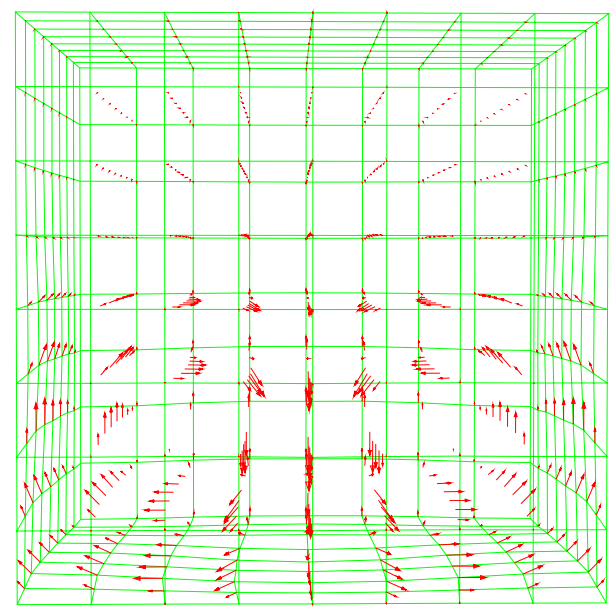

Figure 7: Visualization of the results of a coupled three-dimensional simulation based on an ALEformulation for the electromagnetic field relations. The arrows represent the magnetic flux density vectors. The same geometric conditions as in Figure 6 have been chosen.

\section{CONCLUSIONS}

A fully-coupled three-dimensional simulation of EMF has been presented based on a thermoelastic, viscoplastic, electromagnetic material model incorporated in a large- 
deformation dynamic Lagrangian formulation and Maxwell's equations under quasistatic conditions. To compute the Lorentz forces in high accuracy, an ALE approach for the electromagnetic fields has been chosen. The adaption of the electromagnetic mesh to the moving structure avoids, on the one hand, unphysical oscillations of the computed forces and simplifies, on the other hand, the field equations. In contrast to remeshing strategies this approach preserves the combinatorial structure of the mesh, which allows an effective solution of the arising huge systems of linear equations. To discretize the electromagnetic system, a novel, non-isoparametric version of Nédélec elements is employed. This formulation avoids a bad approximation at material interfaces due to the discontinuity of the normal component of the vector potential, guarantees a Coulomb gauge to decouple the electromagnetic field relations, and avoids a loss of accuracy in case of large mesh deformation, which would arise in an isoparametric context. A comparison of simulations based on the methods presented here to experimental data represents work in progress.

\section{References}

[1] W. H. Gourdin. Analysis and assessment of electromagnetic ring expansion as a high-strain rate test. J. Appl. Phys., 65, 411, (1989).

[2] W. H. Gourdin, S. L. Weinland and R. M. Boling. Development of the electromagnetically-launched expanding ring as a high strain-rate test. Rev. Sci. Instrum., 60, 427, (1989).

[3] N. Takata, M. Kato, K. Sato and T. Tobe. High-speed forming of metal sheets by electromagnetic forces. Japan Soc. Mech. Eng. Int. J., 31, 142, (1988).

[4] G. Fenton and G. S. Daehn. Modeling of electromagnetically formed sheet metal. J. Mat. Process. Tech., 75, 6-16, (1998).

[5] C. Beerwald, A. Brosius and M. Kleiner. Determination of flow stress at very high strain-rates by a combination of magnetic forming and FEM calculation. In Proceedings of the International Workshop on Friction and Flow Stress in Cutting and Forming (CIRP). ENSAM - Paris, (2000).

[6] A. Brosius, T. Chanda, M. Kleiner and T. Svendsen. Finite-element modeling and simulation of material behavior during electromagnetic metal forming. In Proceedings of the 6th International ESAFORM Conference on Material Forming 28.-30. April 2003, Italy, pages 971-974. ESAFORM 2003, (2003).

[7] B. Svendsen and T. Chanda. Continuum thermodynamic modeling and simulation of electromagnetic forming. Technische Mechanik, 23, 103-112, (2003).

[8] B. Svendsen and T. Chanda. Continuum thermodynamic formulation of models for electromagnetic thermoinlastic materials with application to electromagnetic metal forming. Cont. Mech. Thermodyn., 17, 1-16, (2005). 
[9] M. Stiemer, J. Unger, H. Blum and B. Svendsen. Algorithmic formulation and numerical implementation of coupled multifield models for electromagnetic metal forming simulations. Int. J. Numerical Methods in Engineering, (2006). Accepted.

[10] B. Jiang, J. Wu and L. A. Povinelli. The origin of spurious solutions in computational electromagnetics. Journal of computational physics, 125, 104-123, (1996).

[11] J. C. Nédélec. Mixed Finite Elements in $\mathbb{R}^{3}$. Numerische Mathematik, 35, 315-341, (1980).

[12] J. C. Nédélec. A New Family of Mixed Finite Elements in $\mathbb{R}^{3}$. Numerische Mathematik, 50, 57-81, (1986).

[13] F. Moon. Magnetic interactions in solids. Springer-Verlag, (1980).

[14] J. D. Jackson. Classical Electrodynamics. John Wiley and Sons, (1987).

[15] B. Svendsen. On the modeling of anisotropic elastic and inelastic material behaviour at large deformation. Int. J. Solids Structures, 38, 9579-9599, (2001).

[16] S. Reese and B. Svendsen. On the modeling of internal variables as structure tensors in anisotropic, finite-deformation inelasticity. J. de Physique IV, 14, (2003).

[17] P. Rosakis, A. J. Rosakis, G. Ravichandran and J. Hodowany. A thermodynamic internal variable model for the partition of plastic work into heat and stored energy in metals. J. Mech. Phys. Solids, 48, 581-607, (2000).

[18] C. Teodosiu. Dislocation modeling of crystalline plasticity. In C. Teodosiu, editor, Large plastic deformation of crystalline aggregates, number 376 in CISM, pages 2180, (1997).

[19] U. F. Kocks and F. Mecking. The physics and phenomenology of strain hardening: the FCC case. Prog. Mat. Sci., 98, 171-273, (2003).

[20] H. J. Frost and M. F. Ashby. Deformation mechanism maps. Pergamon Press, (1982).

[21] J. C. Simo and T. J. R. Hughes. Computational Inelasticity. Number 7 in Springer Series on Interdisciplinary Applied Mathematics. Springer, (1998).

[22] M. Schinnerl, J. Schöberl, M. Kaltenbacher and R. Lerch. Multigrid Methods for the 3D Simulation of Nonlinear Magneto-Mechanical Systems. IEEE transactions magnetics, 38, 1497-1511, (2002).

[23] T. J. R. Hughes. The Finite Element Method. Prentice-Hall, (1987).

[24] M. Badelt, C. Beerwald, A. Brosius and M. Kleiner. Process Analysis of Electromagnetic Sheet Metal Forming by Online-Measurement and Finite Element Simulation. In Proceedings of the 6th International ESAFORM Conference on Material Forming, 28.-30. April 2003, Italy, pages 123-126. ESAFORM 2003, (2003).

[25] N. Jones. Structural Impact. Cambridge University Press, (1989). 